Fazit: Bei Patienten mit Asthma und Rhinitis kann die Applikation von nasalen Glukokortikoiden und oralen Antihistaminika schwere Asthmaexazerbationen verhindern. Um entsprechende Therapieempfehlungen aussprechen $\mathrm{zu}$ können, sollten die Ergebnisse jedoch noch in einer prospektiven randomisierten Studie bestätigt werden. $\quad b k$

Corren J et al. Rhinitis therapy and the prevention of hospital care for asthma: a case-control study. J Allergy Clin Immunol 2004; 113: 415-9

\title{
Glukokortikoidsprays richtig inhalieren
}

\author{
Wirksamkeit und Verträglichkeit nasaler Glukokortikoide hängen \\ auch davon ab, wie das Spray appliziert wird. Doch gibt es bisher \\ keine „Leitlinie“ für eine optimale Inhalationstechnik. US-amerika- \\ nische Wissenschaftler sammelten nun Tipps aus der Literatur und \\ entwickelten daraus die ideale Applikationstechnik für Patienten.
}

$\mathrm{V}$ ergleicht man die Anweisungen verschiedener Hersteller zur Applikation nasaler Glukokortikoidsprays, so findet man deutliche Unterschiede. Beispielsweise variieren die Empfehlungen bezüglich der vorherigen Applikation eines abschwellenden Medikaments, wenn der Patient sehr viel Sekret produziert oder die Nasenschleimhaut geschwollen ist. Auch sollte der Kopf in der einen Anweisung nach vorne, in der anderen nach hinten geneigt werden. Weitere Unterschiede betreffen die „Nachbereitungsphase“, für die ein Luftanhalten über einige Sekunden oder das Nachhintenlegen des Kopfes empfohlen wird.

Vor diesem Hintergrund sichtete ein Team der American Academy of Otolaryngology die Literatur sowie die Empfehlungen der Hersteller, um eine evidenzbasierte Applikationstechnik zu entwickeln. Aus zunächst 121 Publikationen wurden 29 methodisch geeignete Studien ausgewählt und ausgewertet. Insgesamt stellte sich keine einzelne Applikationstechnik als eindeutig überlegen heraus. In der Regel wird bei jeder Applikation der größte Teil des Wirkstoffs im vorderen unteren und mittleren Nasenraum deponiert, von wo aus die Substanzen mit Hilfe der - dort allerdings nur spärlich vorhandenen - Zilien weiter ins Naseninnere transportiert werden. Die vorherige Applikation von abschwellenden Substanzen mag die Verteilung im mittleren Nasengang verbessern, als Routinemethode ist sie nicht geeignet. Auf jeden Fall sollten die Patienten nicht direkt das Nasenseptum ansprühen. Zwar liegen dazu keine kontrollierten Studien vor, doch scheint damit ein erhöhtes Risiko für Nasenbluten verbunden zu sein.

Fazit: Nasale Glukokortikoidsprays lassen sich einfach applizieren. Für Patienten verständliche und praktikable Empfehlungen sind im Kasten wiedergegeben. $b k$

Benninger MS et al. Techniques of intranasal steroid use. Otolaryngol Head Neck Surg 2004; 130: 5-24

Nasale Applikation Step by Step

_ Falls nötig, Nase schneuzen.

_ Zum Sprayen ins linke Nasenloch möglichst die rechte Hand verwenden - und umgekehrt.

— Sprayöffnung in das Nasenloch einführen.

— Sprayöffnung nicht auf die Nasenscheidewand, sondern eher nach außen richten

— Kopf in einer aufrechten Position halten.

- Spray nach Angaben des Herstellers bedienen, die Anzahl der Spraystöße richtet sich nach den Anweisungen des Arztes.

— Während des Sprayvorgangs behutsam einatmen oder die Nase hochziehen.

— Durch die Nase ausatmen. 